\title{
AN IMPROVED METHOD OF RATING TUNING FORKS.
}

\author{
BY JOHN O. REED.
}

ATHOUGH the tuning fork has long been used as a means of A measuring small intervals of time, yet a cheap, convenient and accurate method of rating the fork has remained a thing much to be desired. Of the various methods proposed, the most successful have generally employed the stroboscopic principle in some form or other. Of these the method first described by Michelson ${ }^{1}$ is unquestionably the most simple and convenient, although attended with experimental difficulties which materially limit its usefulness and detract from the accuracy of which it is otherwise capable. 'In the effort to obviate these difficulties the writer has resorted to a number of devices, some of which are, it is thought, sufficiently successful to warrant their description as a distinct method.

In the method as described by Michelson, the standard fork is compared with an auxiliary fork by counting beats; the auxiliary fork is driven electrically and is furnished with a mirror, in which is viewed the flash of a Geissler tube ; this tube is actuated by an induction coil whose primary circuit is periodically closed by a pendulum of known period. When the fork is at rest the flashes are seen at one place and appear at rest. When the fork is made to vibrate the flashes form a stroboscopic procession back and forth across the field of view, and from the number of flashes needed to complete the cycle the frequency of the fork is readily determined, provided its value is approximately known.

The difficulty in securing exact periodicity in the flashes of the tube forms a most serious obstacle in the use of the method, as originally described. Michelson points out that if the intervals between the flashes differ by 0.002 of a second, the method becomes

${ }^{1}$ A. A. Michelson, Philos. Magazine, Vol. 15 (5), p. 84, 1883 . 
impossible for a fork of frequency I $28 \mathrm{v}$. s. He accordingly devotes much attention to the mercury contact in order to make the flashes as nearly isochronous as possible. The sources of error due to this contact are numerous. First, the mercury globule must be exactly in the center of the arc of the pendulum, an adjustment difficult to get and troublesome to maintain. Again if the globule have any appreciable thickness in the direction of the moving point, there is an interval of time between the make and break of the primary current, sufficient to give rise to a system of double flashes of varying intensity. These become extremely confusing to the observer trying to follow the stroboscopic procession across the field of view, and in many cases it is impossible to decide in which direction the flashes are moving. Oscillatory discharges of the coil are also possible, and the flashes become very complex and misleading.

By properly adjusting the resistances in the primary and secondary circuits, and by making the globule thin and flat, the abovementioned difficulties may be largely obviated, but there remain more serious objections; first, the time of discharge of the coil through the tube is not a definite interval, much less is it amenable to control or regulation; again, the mercury globule soon becomes coated with a film of oxide, even when weak currents are used, and this film drags after the contact point, forming a minute tail which prolongs the time of break in some cases as much as one or two thousandths of a second and is always irregular and uncertain in its action. Now, the fundamental assumption in the application of the stroboscopic method is that the intermittent illumination shall be strictly isochronous and that the time of illumination shall be vanishingly small as compared with the period of the motion to be studied, in order that the body may not move sensibly during the illumination. Consequently an illumination that would be entirely satisfactory for a fork of frequency 64 , would be inadequate for work upon a fork of frequency 128 or 192 , since in the latter case the flashes would be spread out into broad bands of three times the width of the same flashes as seen in the mirror of fork 64 .

It is evident, therefore, that a system of illumination intended for use in stroboscopic work must not only be isochronous to any 
required degree of accuracy, but if it is to find general application, it must also be entirely under control as regards the duration of the illumination, and this duration must be capable of adjustment within considerable limits. From the above considerations it is clear that the flash of a Geissler tube is but poorly adapted to the stroboscopic study of vibratory motions of even moderate frequency, and a new source of intermittent illumination must be provided. A satisfactory substitute is found in the following arrangement.

A rod $R_{1}, 8 \mathrm{~mm}$. in diameter and $100 \mathrm{~cm}$. long, hung from two short pieces of clock spring, $a a$, carries on its lower end a large lens-shaped mass of lead, $L$, and four adjustable weights I, 2, 3, 4, the whole forming a free pendulum of adjustable period. Below the middle of the rod is clamped a ring to which is attached a shortarm $l$, bearing at its outer end a small needle $n$. Against this rests a short piece of fine glass tube $t$, about 0 . I $\mathrm{mm}$. in diameter and attached normally to a vertical axis $p$ which turns in point centers and carries a plane mirror $m$. As the pendulum swings in a plane normal to the plane of the paper, the mirror is caused to rotate about the vertical axis $p$, through a relatively large arc whose angular amplitude $A$, is given in terms of $a$, the angular amplitude of the pendulum, by the relation $a R=A r$ or $A=a R / r$, where $R$ and $r$ represent the effective radii of motion of the pendulum and mirror respectively at the point of contact of the tube and needle. The tube is held against the needle by means of a small drop of oil at

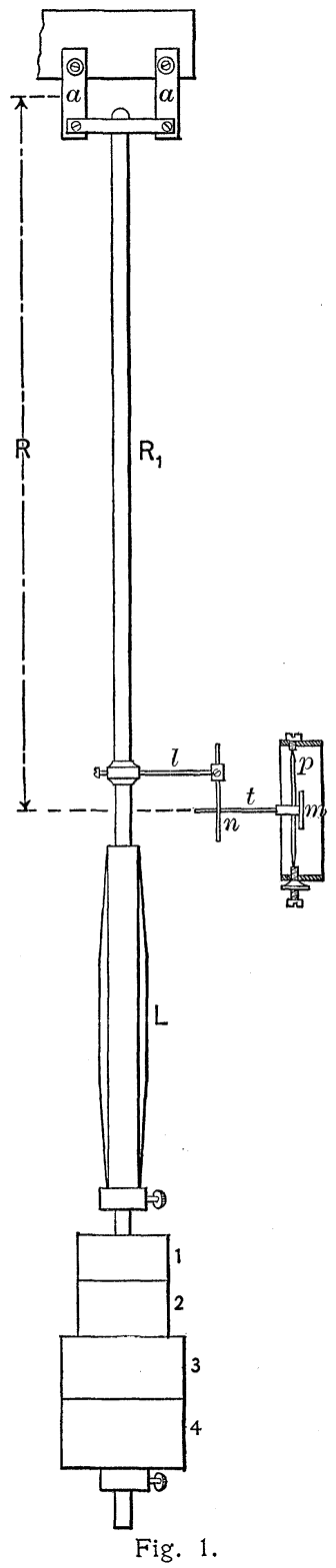


the point of contact, the viscosity of the oil being sufficient to carry the mirror on its return swing.

If now there be thrown upon the mirror $m$ a beam of light from any convenient source, we have a moving spot of light which may be given any desired linear velocity, and whose successive vibrations are isochronous to a high degree of accuracy. The source of light is a brightly illuminated slit, shown at $s$ (Fig. 2) from which the light is focussed at $s^{\prime}$ by the lens $l$ after reflection at the mirror $m . T$ is an Abbé autocollimating telescope, having a totally reflecting prism in the focal plane of the eyepiece, by means of which the light entering the window at $s^{\prime}$ is thrown directly into the axis of the telescope and leaves the objective in

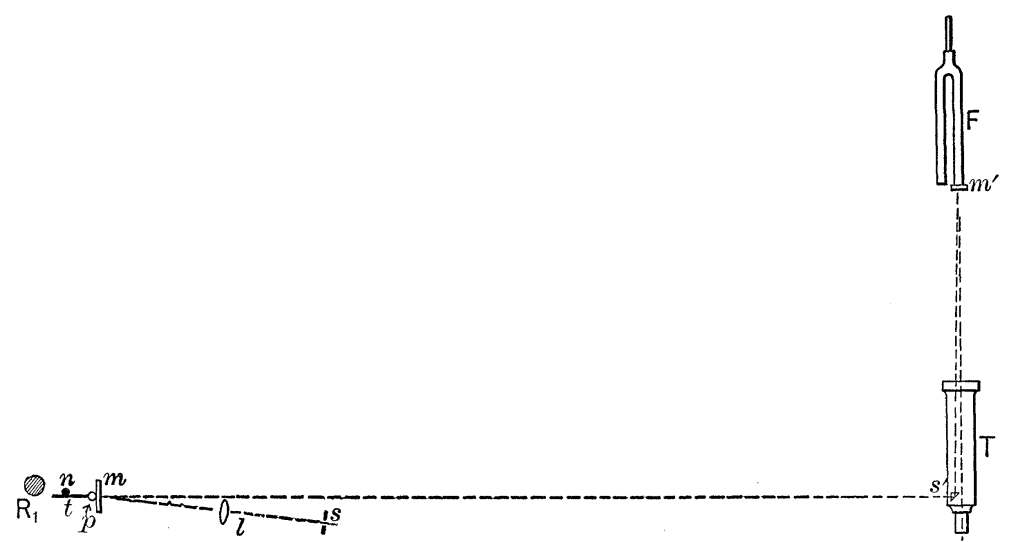

Fig. 2.

parallel rays. Directly in front of the prism is an adjustable slit $s^{\prime \prime}$ (not shown in the figure), thus allowing the telescope to serve both as collimator and observing telescope. The parallel rays from the telescope strike the mirror $m^{\prime}$ normally, and return to form an image of the slit $s^{\prime \prime}$ in the focal plane of the telescope. So long as both pendulum and fork are at rest there is seen in the field of the telescope a single bright image of the slit. If the pendulum be set in motion this image flashes brightly each time the image $s^{\prime}$ sweeps past the small window in the telescope, the duration of the flash with a given pendulum depending upon the width of the window, the width of the image $s^{\prime}$, the distance $m s^{\prime}$, and the ratio $R / r$. 
When the fork is set in motion the image in the telescope becomes a stroboscopic procession of images moving across the field, each one so sharp and distinct that coincidences with the cross hair of the telescope may be observed or approximate coincidences estimated to a fifth of the width of a slit image without difficulty.

In practice the free pendulum is rated in terms of the standard clock at the same time that the observations are made on the fork, one observer being able to carry on both sets of readings by working alternately upon first the one and then the other. By determining the period of the pendulum at the time of the observations upon the fork, corrections for effects of temperature upon the pendulum are obviated. The rating is effected by the method of coincidences as employed by Mendenhall ${ }^{1}$ in his work upon the determination of $g$. The coincidences are observed by viewing the flash of a Geissler tube marking seconds, in a system of mirrors, one fixed and the other attached to the free pendulum. The "coincidence" occurs when the composite image of the flash appears as an unbroken line. To this end the pendulum carries a small mirror about $3 \mathrm{~cm}$. wide and $6 \mathrm{~cm}$. long, and near it in the same vertical plane, is placed a fixed mirror of the same size, so adjusted that the image of the capillary part of the Geissler tube appears as one straight piece when seen in the mirrors with the pendulum at rest. It is necessary to take the coincidences with the pendulum of the clock always going in the same direction, in order to avoid the error due to any slight eccentricity in the position of the mercury globule.

Under ordinary circumstances the period of the pendulum can be determined to hundred-thousandths of a second in twenty minutes, usually not more than five coincidences being necessary. During the intervening intervals a sufficient number of cycles of the fork can be counted to give all the data needed to determine its frequency so that the entire process may be completed in half an hour. In laboratory practice it is customary to require the student to make three determinations and take the mean. For this purpose three different pendulums are arranged by making suitable combinations of the adjustable weights I, 2, 3, 4 shown in Fig. I, so 
that for each pendulum a workable cycle of flashes from the fork may be secured. The best results are obtained by using cycles of not less than five nor more than ten flashes per cycle. The computation is as follows :

Let $N$ be the frequency of the fork to be rated.

Let $t_{1}, t_{2}, t_{3}$ be the periods of the pendulums $\mathrm{I}, 2,3$, where $t_{1}<t_{2}<t_{3}$.

Let $n_{1}, n_{2}, n_{3}$ be, to the nearest integer, the number of complete vibrations made by the fork in times $t_{1}, t_{2}, t_{3}$.

Let $a_{1}, a_{2}, a_{3}$ be the number of flashes per cycle made by the fork with pendulums I, 2, 3 ; where $5<a<$ IO.

Let $c_{1}, c_{2}, c_{3}$ be the reciprocals of $a_{1}, a_{2}, a_{3}$ respectively. Then

$$
\begin{aligned}
& N t_{1}=n_{1} \pm c_{1} \\
& N t_{2}=n_{2} \pm c_{2} \\
& N t_{3}=n_{3} \pm c_{3} .
\end{aligned}
$$

If now $\left(t_{3}-t_{1}\right)<\frac{\mathrm{I}}{2 N}$ then $n_{1}=n_{2}=n_{3}=n$, and we have

$$
N=\frac{n \pm c_{1}}{t_{1}}=\frac{n \pm c_{2}}{t_{2}}=\frac{n \pm c_{3}}{t_{3}}
$$

If $N$ be approximately known, $i . e$., to the nearest integer, then $n$ may be set equal to $N t$. If $N$ be wholly unknown the graphical method may be employed to determine its approximate value.

The proper sign to be chosen for $c$ in the three equations is shown from the following consideration: The free pendulum as it swings decreases in period very slightly as the amplitude decreases ; this decrease in period is evidenced by a slight but unmistakable variation in $a$, the number of flashes per cycle in each individual case, and consequently by a corresponding variation in $c$, the fraction of a swing by which the fork exceeds or falls short of an integral number of swings in time $t$. Now as $t$ decreases this excess will diminish or the deficit will increase; therefore if $a$ is found to increase during the experiment $c$ is positive; if $a$ is found to decrease, $c$ is negative.

In making a determination the apparatus is first carefully adjusted until the spot of light falls exactly on the window of the 
telescope when the pendulum is at rest, and the image of the slit is very bright. The pendulum is then started, the fork set in vibration and the cycle of flashes roughly determined. If necessary the pendulum must be adjusted by moving the small weights at the lower end until the number of flashes per cycle lies between 5 and Io. For convenience of reference a small sketch of the pendulum showing position of weights is usually appended. The pendulum observations are then started by taking three or four coincidences in quick succession, to get its cycle roughly, after which the coincidences may be taken in groups of three at intervals of five minutes or more as the work proceeds. Only one of each group is used, the others being merely to insure against possible failure to keep track of the pendulum. In the intervals between the observations on the pendulum the fork is bowed and the coincidence of the flash with the cross wire of the telescope awaited; when this occurs the flashes are counted continuously, the observer making note of the coincidences, underscoring those especially sharp, as long as the fork will run, or until the images can no longer

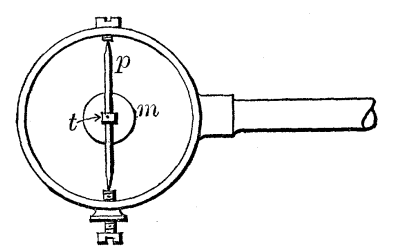

Fig. 3. be seen sharply separated. The fork is bowed again and the observations repeated. In the computation the mean value of $a$ is to be taken, and exceptionally sharp coincidences are to be assigned greater relative importance in determining the value of $\alpha$; from this the value of $c$ is computed.

It is well to note the time of beginning each set of observations upon the fork, as in this way the varying value of $a$ may be readily followed.

The following example of a complete determination will render the method clear: 
Pendulum gaining. 6 in 64; better 14 in 150. January 2, 1898.

\begin{tabular}{|c|c|c|c|c|}
\hline Transits. & Seconds. & Vibrations. & Period. & Fork bowed. \\
\hline 30211 & \multirow{3}{*}{64} & \multirow{3}{*}{70} & \multirow{3}{*}{0.914} & \multirow{3}{*}{$\begin{array}{l}6<\text { a }<7 \\
\text { nearer } 6 .\end{array}$} \\
\hline 0315 & & & & \\
\hline 0545 & & & & \\
\hline 0711 & \multirow[t]{3}{*}{300} & \multirow[t]{3}{*}{328} & \multirow[t]{3}{*}{0.9146} & \multirow{3}{*}{$\begin{array}{l}3,07, I I \\
6,44,88\end{array}$} \\
\hline 1421 & & & & \\
\hline 1525 & & & & \\
\hline 1651 & 880 & 962 & 0.91476 & $6,44,63,88$ \\
\hline 2901 & \multirow[t]{3}{*}{1610} & \multirow[t]{3}{*}{1760} & \multirow[t]{3}{*}{0.91477} & \\
\hline 3027 & & & & $6,25,44,88$ \\
\hline 3131 & & & & ................... \\
\hline 3549 & \multirow[t]{2}{*}{2018} & \multirow[t]{2}{*}{2206} & \multirow[t]{2}{*}{0.914778} & $3,16,5 I$ \\
\hline 3819 & & & & $6,3^{I}, 43$ \\
\hline 3945 & \multirow[t]{2}{*}{2254} & \multirow[t]{2}{*}{2464} & \multirow[t]{2}{*}{0.914773} & 86 \\
\hline 4341 & & & & $6,37,74$ \\
\hline 4507 & 2676 & 2816 & 0.914773 & $6,43,49,98$ \\
\hline \multicolumn{5}{|c|}{$t_{1}=0.91477 \mathrm{sec}}$. \\
\hline
\end{tabular}

Computation of $a_{1}$.

\begin{tabular}{|c|c|c|c|}
\hline Flashes. & Cycles. & $a$. & \\
\hline 1st set. 44 & 7 & 6.285 & $6.285 \times 9=56.565$ \\
\hline 63 & 10 & 6.300 & 6.300 \\
\hline \multirow[t]{3}{*}{25} & \multirow[t]{3}{*}{4} & \multirow[t]{3}{*}{6.25} & 6.250 \\
\hline & & & 11) 69.115 \\
\hline & & & $a=6.2^{83}$ \\
\hline \multirow{7}{*}{$\begin{array}{r}2 d \text { set. } 31 \\
43 \\
49 \\
37\end{array}$} & 5 & 6.200 & 6.200 \\
\hline & 7 & 6.1428 & $6.1428 \times 4=24.5712$ \\
\hline & 8 & 6.125 & $6.125 \times 3=18.375$ \\
\hline & 6 & 6.166 & $6.1666 \times 3=18.4998$ \\
\hline & & & 11) 67.6460 \\
\hline & & & $a=6.1487$ \\
\hline & & & $a_{1}=6.2158$ \\
\hline
\end{tabular}

$a_{1}$ decreasing, hence $c_{1}$ is negative.

$$
\begin{aligned}
& c_{1}=-0.16089 \\
& n=30 . \\
& t_{1}=0.91477 \mathrm{sec} . \\
& N=\frac{29.839 \mathrm{II}}{0.91477}=32.6 \mathrm{I} 9 .
\end{aligned}
$$


A second fork gave for ten different determinations, by different persons on different days, the following values:

Temperature.
$13.0^{\circ}$
$17.5^{\circ}$
$17.7^{\circ}$
$18.8^{\circ}$
$19.5^{\circ}$
$21.0^{\circ}$
$22.7^{\circ}$
$23.0^{\circ}$
$23.0^{\circ}$
$23.4^{\circ}$

$\begin{array}{cc}N_{t} & N_{20} \\ 32.493 & 32.468 \\ 32.475 & 32.466 \\ 32.473 & 32.465 \\ 32.472 & 32.468 \\ 32.469 & 32.467 \\ 32.464 & 32.467 \\ 32.458 & 32.468 \\ 32.457 & 32.469 \\ 32.455 & 32.467 \\ 32.456 & 32.469\end{array}$

Reduction to $20^{\circ} \mathrm{C}$. has been made by the use of Koenig's formula, ${ }^{1} \Delta N=\frac{-N}{8943}$ per degree centigrade.

For forks of higher frequency the requirements are greater accuracy in the adjustment of the light upon the telescope slit when the pendulum is at rest, more powerful illumination of the slit and greater velocity of the moving image. The last requirement is met by increasing the ratio $R / r$, and the distance $m s^{\prime}$. With a slit illuminated by an acetylene flame or an arc light, no difficulty is experienced in reducing the duration of the flash to $0.0000 \mathrm{I}$ of a second, a period sufficiently short to show the slit image in the telescope, sharp and clear, when reflected from the mirror of a fork making 256 double vibrations per second, even in the middle of its swing. This is readily seen from the following considerations: Assume the amplitude of the motion of the slit image as seen in the telescope to be $5 \mathrm{~mm}$; then the maximum velocity of the image is $8,050 \mathrm{~mm}$. per second. Also assuming the limit of the resolving power of the eye to be $0.073 \mathrm{~mm}$. at the distance of distinct vision, we have for the duration of the flash

$$
t=\frac{0.073}{8050}=0.000009 \text { second }
$$

In case it is not desirable to attach a mirror to the fork under investigation, Michelson's second method may be applied; that is the flashes from the pendulum may be received directly into the objec-

${ }^{1}$ Koenig, Wied. Annalen, I880, N. F., 9, p. 413. 


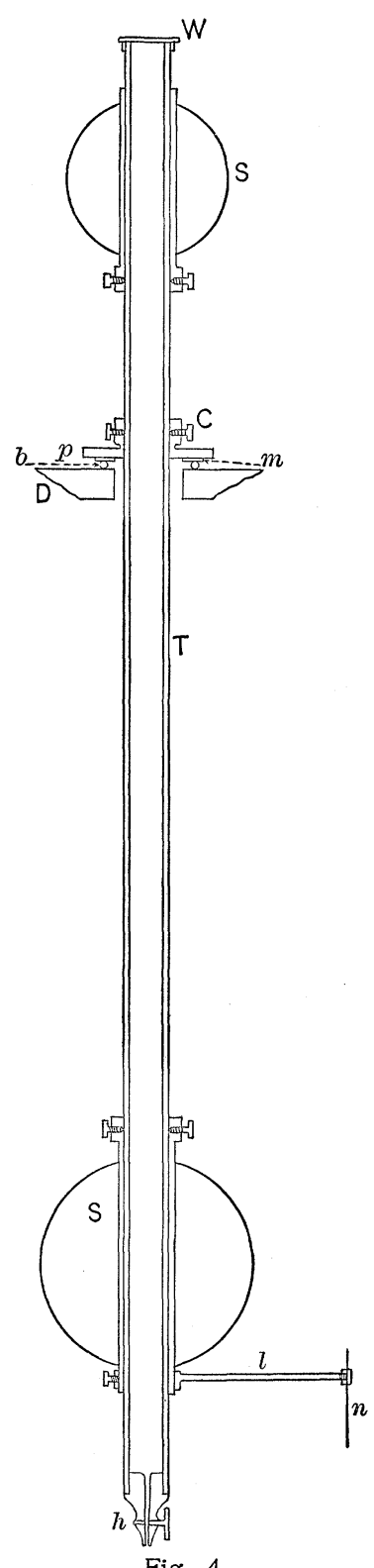

tive of a low power microscope in front of which is placed the fork with one prong sharply focussed so as to fill half the field when at rest. The flashes will then give a stroboscopic procession of shadows instead of images of the slit as before. Even by this method forks may be rated, whose frequency would entirely preclude the use of the Geissler tube.

For the purposes of an investigation of which an account will appear later, it became necessary to vary the period of the flashes continuously and by any desired amount. To this end a special form of free pendulum was devised, the main features of which are shown in Fig. $4 .^{1}$ A piece of bicycle tubing $T, 2.5 \mathrm{~cm}$. in diameter and $100 \mathrm{~cm}$. long is fitted with an adjustable collar $C$, to which is attached a brass plate $p, 7 \mathrm{~mm}$. thick. On the under side of this plate are fastened two small plates $\mathrm{mm}^{\prime}$ of hardened steel, each $3 \mathrm{~mm}$. thick and having their under surfaces ground plane. These plates rest upon two small bicycle balls $\frac{3}{32}$ of an inch in diameter, which in turn rest in two small depressions in the bed plate $D$. At either end of the tube $T$ are clamped spheres of lead $S S^{\prime}$ of 7 and io kilograms mass respectively. By means of set screws these spheres may be given any desired position on the tube.

At the lower end of the tube is fitted a steel plug and stopcock $h$, by means

1 This pendulum was made by Mr. R. H. Miller, instrument maker to the university, to whose practical suggestions and skillful workmanship much of its admirable performance is due. 
of which a quantity of mercury held in the tube may be varied at will. A small collar holds the horizontal arm and the needle for actuating the rotating mirror as shown in Fig. I. The bed plate $D$ rests upon three short pointed feet which are held in place upon a steel plate on the supporting frame by the familiar hole and slot arrangement. The whole is supported by a rigid frame of suitable height, firmly clamped to a stone pier.

By adjusting the relative positions of the supporting plate and the two lead spheres, the period of the pendulum may be varied within wide limits. In practice the period is kept approximately either 0.5 second or one second for a single swing. In the latter case the system with rotating mirror attached will vibrate about three hours and still retain sufficient amplitude to permit of satisfactory observations. With smaller balls the duration of the motion may be made even longer.

As will readily be seen the variation of the period in order to obtain a workable cycle of flashes may now be made to any degree of accuracy and also while the pendulum is in motion. To this end mercury is poured into the tube until the period is slightly longer than desired. The apparatus being adjusted the pendulum is set in motion and the cycle observed. If not satisfactory a tap of the pencil upon the stopcock $h$, allow's a fine stream of mercury to flow out into a convenient receptacle, and the flashes are observed until a workable cycle is secured when a second tap of the pencil closes the stopcock. In this way the pendulum may be "tuned" to any desired period with great accuracy. The enclosed column of mercury serves the additional purpose of rendering the pendulum much less sensitive to changes of temperature, and in case it were deemed desirable, practically complete compensation could be secured in this way. In such case the variation of the period may be effected by placing small weights upon the small cap $w$, at the upper end of the pendulum.

By the use of the above pendulum the following values were sesured for the frequency of a fork marked $5 \mathrm{I} 2 \mathrm{v}$. s. using the mirror and flash method. 
Temperature.

$$
20.2^{\circ}
$$

$21.0^{\circ}$

$22.3^{\circ}$

$20.8^{\circ}$

$23.0^{\circ}$

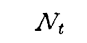

256.019

255.993

255.960

255.995

255.934
$N_{20}$

256.025

256.022

256.026

256.018

256.020

From the above values it is evident that the method is applicable through quite a range of frequency. In as much as the correction for temperature increases as the frequency increases, it is evident also that if any accurate results are to be obtained with forks of high frequency, the question of the exact temperature of the fork and the means of keeping the temperature constant become fundamentally important. For a fork of frequency 256 , the temperature correction is 0.0286 per degree Centigrade; hence an error of $0.3^{\circ} \mathrm{C}$. in the temperature of the fork would account for the maximum outstanding difference in the series of values given above. Under ordinary circumstances the temperature of the fork can best be determined by attaching it to some large mass of metal, with which the thermometers may be brought into thermal contact either by means of mercury cups or by packing the bulbs in tin foil. By enclosing the fork in a hollow cylinder of metal with walls some centimeters thick it is admirably shielded from external temperature effects and its exact temperature may be very accurately determined. To the problem of rating a fork so thermally shielded the method lends itself most readily; only a single tube closed by glass plates being needed for the ingress and egress of the beam of light. The results thus far obtained seem to justify the assumption that with a fork so shielded the method will give the frequency constant in third place of decimals.

For convenience of reference a partial bibliography of the subject is appended.

Lissajous, C. Rendus, I855, xli, 816. (Lissajous' curves.)

Poske, Pogg. Ann., I 874, clii., 449.

Kayser, H., Wied. Ann., N. F., 8, 444, I879. (Temperature effect.)

König, R., Wied. Ann., N. F., 9, 394, I880. (Stimmgabeluhr.)

McLeod and Clarke, Phil. Transactions, i 880, p. I. (Stroboscopic method.)

Lord Rayleigh, Phil. Trans., I883. (Phonic wheel.) 
Michelson, A. A., Phil. Mag., I 5 (5), I883, p. 84.

Weber, R., Jour. de Physique, II, 3, p. 535, I885. (Modification of Siren.)

Mayer, A. M., Phil. Mag., 21 (5), p. 286. (Graphical method.) V. von Lang, Sitz. ber. Ak. z. Wien., r886. (Hipp's chronoscope.)

R. von Oppolzer, Anz. d. K. Akad. Wissensch. zu Wien., I886, p. 82. (Villarceau's regulator.)

Jones, J., Phil. Mag., 27 (5), p. 349, I889. (Application of Lissajous' figures.)

Gregory, W. G., Phil. Mag., 28 (5), p. 49I, I 889. (Electrical driving.)

Ewald, J. R., Durch Luft- oder Wasserstrom bewegte Stimmgabeln, Arch. f. d. gas. Physiologie, 44, I 889, p. 555.

Leman, A., Normalstimmgabeln d. Phys.-Tech. Reichsanstalt, etc., Zeitschr. f. Inst., I890, pp. 77, I70 and 197.

Heerwagen, ., Schwingungsgesetze d. Stimmgabel u. d. Electromagnetische Anregung., Diss. Dorpat, I89I.

Schuller, Alois, Verwendung v. Stimmgabeln u. eine neue Art zu Stimmen, Math. u. Naturw. Berichte aus Ungarn., XII., I894, p. I I 9 .

University of Michigan, AnN Arbor, March, I9oI. 